\title{
Monogenic Binary Coding: An Efficient Local Feature Extraction Approach to Face Recognition
}

\author{
Meng Yang, Student Member, IEEE, Lei Zhang, Member, IEEE, \\ Simon C. K. Shiu, Member, IEEE, and David Zhang, Fellow, IEEE \\ Dept. of Computing, The Hong Kong Polytechnic University, Hong Kong, China
}

\begin{abstract}
Local feature based face recognition (FR) methods, such as Gabor features encoded by local binary pattern, could achieve state-of-the-art FR results in large-scale face databases such as FERET and FRGC. However, the time and space complexity of Gabor transformation are too high for many practical FR applications. In this paper, we propose a new and efficient local feature extraction scheme, namely monogenic binary coding (MBC), for face representation and recognition. Monogenic signal representation decomposes an original signal into three complementary components: amplitude, orientation and phase. We encode the monogenic variation in each local region and monogenic feature in each pixel, and then calculate the statistical features (e.g., histogram) of the extracted local features. The local statistical features extracted from the complementary monogenic components (i.e., amplitude, orientation and phase) are then fused for effective FR. It is shown that the proposed MBC scheme has significantly lower time and space complexity than the Gabor-transformation based local feature methods. The extensive FR experiments on four large scale databases demonstrated the effectiveness of MBC, whose performance is competitive with and even better than state-of-the-art local feature based FR methods.
\end{abstract}

Keywords: monogenic signal analysis, monogenic binary coding, face recognition, LBP, Gabor filtering

Copyright (c) 2012 IEEE. Personal use of this material is permitted. However, permission to use this material for any other purposes must be obtained from the IEEE by sending a request to pubs-permissions@ieee.org.

Meng Yang, Lei Zhang, Simon C. K. Shiu, and David Zhang are with the Dept. of Computing, The Hong Kong Polytechnic University, Hong Kong, China. Corresponding author: Lei Zhang, Email: cslzhang@comp.polyu.edu.hk. This work is supported by the HK RGC PPR grant (PolyU5019-PPR-11). 


\section{Introduction}

Automatic face recognition (FR) has been extensively studied in the past two decades, yet it is still an active research topic, partially because of its many challenges in practice such as uncontrolled environments, occlusions and variations in pose, illumination, expression and time. FR has a wide range of applications, including entertainment, smart cards, information security, law enforcement, access control and video surveillance [1][2]. Various methods have been proposed for facial feature extraction and classification, among which the representatives include subspace learning (e.g., Eigenface [5], Fisherface [6], Laplacianfaces [37], 2DPCA-based discriminant analysis [17]), discriminative models for age invariant FR [3][4], Gabor feature based classification (GFC) [7], local binary pattern (LBP) and its variants [8][9][10][11][22][23], local appearance and shape based methods for 3D FR [24][38], the recently developed sparse representation based methods [29][30][35][39][40], and the like.

Unlike many appearance-based FR methods, which are either holistic feature based (e.g., Eigenface and Fisherface) or local feature based (e.g., GFC), the adoption of LBP in FR [8] triggers the use of local statistical feature (LSF) in the FR field. Fig. 1 shows the pipeline of LSF based FR. One can see that LSFFR methods have two main phases: statistical histogram feature extraction and statistical feature combination. Histogram feature extraction could be further divided into three steps: feature map generation (e.g., original image, Gabor feature), pattern map coding (e.g., LBP) and sub-region histogram computing. Almost all the LSF-FR methods [8][9][10][11][22][23] have similar procedures of sub-region histogram computing (i.e., extracting the statistical information of pattern feature in each sub-region), which shows certain robustness to local deformations (e.g., pose, expression, and occlusion) of face images. However, different schemes of feature map generation and pattern map coding lead to different LSF-FR methods.

The well-known LBP operator [8] directly encodes the image intensity to extract image local pattern features. In order to overcome the sensitiveness of intensity to image variations (e.g., illumination), Zhang et al. [9] proposed to extract directional Gabor magnitude features at multiple scales, and then apply LBP to the Gabor magnitude maps for robust LSF. The studies of Gabor phase based LSF-FR were conducted in [10-11, 22]. Zhang et al. [10] adopted multi-scale Gabor phase to take the place of Gabor magnitude in [9], and the global and local variations of real part and imagery part of complex Gabor filtering coefficients were encoded in [11]. Recently, Xie et al. [22] utilized XOR (exclusive or) operator to encode the local variation 
of Gabor phase, and then fused Gabor-magnitude local pattern and Gabor-phase local pattern. This scheme achieves very promising FR results.

\section{Inputimage}

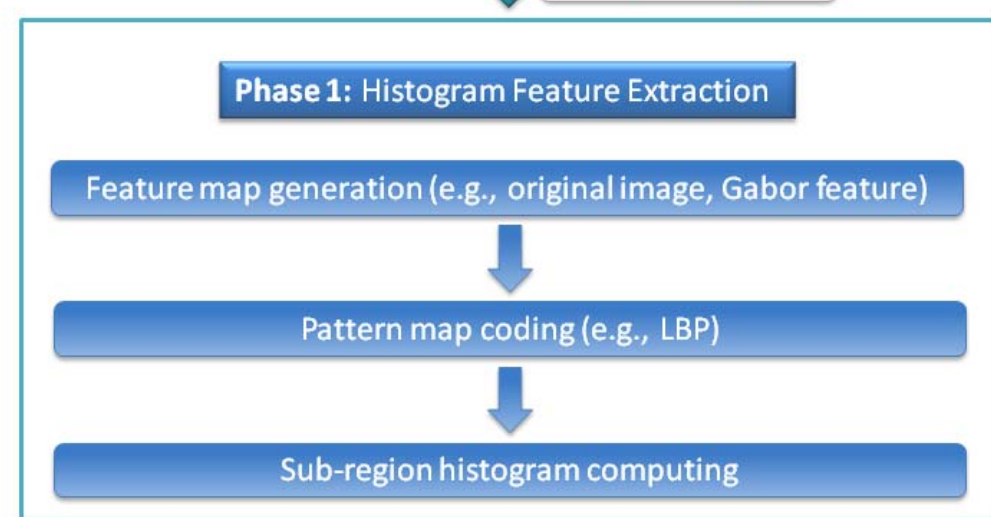

Histogram feature

Phase 2: Feature Combination (e.g., weighting, LDA)

Figure 1: The pipeline of local statistical feature based face recognition (LSF-FR).

For the second phase of LSF-FR, many feature combination methods have been proposed [811][22][26][36]. The most commonly used strategy is weighting the histogram feature extracted in different blocks [8-11]. In [26] and [36] the kernel linear discriminant analysis was used to reduce the dimension of histogram feature, and in [22] a block-based Fisher linear discriminant (BFLD) method was proposed to extract the low-dimensional discriminative features. Compared with weighting based histogram feature combination, the discriminant subspace learning methods [26][36][22] are much more preferred because they can not only improve the feature discrimination, but also greatly reduce the storage space.

Despite the great success of Gabor feature-based LSF-FR methods [9][10][11][22], the expenses of Gabor transformations in these methods are also rather high, including both the computational cost and the storage space, because the Gabor transformations of an image need to be implemented at multiple scales and orientations. For example, 5-scale and 8-orientation Gabor transformations are used in [7][9-11][22]. 
Therefore, for a single image 80 convolutions (40 real convolutions and 40 imagery convolutions) are required to generate the Gabor features (e.g., 40 Gabor magnitude feature maps). The many convolutions and the Gabor feature maps make Gabor feature generation a process of high time and space complexity, preventing its wide acceptance in practical applications.

In our previous work [23], we investigated the use of monogenic signal analysis [12] for LSF-FR, and our proposed monogenic signal decomposition based local feature extraction has much lower time and space complexity than the Gabor filtering based methods [9][11] but leads to competitive performance in FR. As a two-dimensional generalization of one-dimensional analytic signal representation, monogenic signal representation decomposes an image into amplitude, phase, and orientation components, which represent the signal energetic, structural, and geometric information, respectively [12]. Different from Gabor transformation, monogenic signal representation does not use steerable filters to extract multiple-orientation features, and thus it has much lower time (e.g., 3 convolutions on each scale) and space (e.g., 3 feature maps on each scale) complexity than Gabor transformations.

To further exploit the discrimination information embedded in the amplitude, phase and orientation components of monogenic signal representation, in this paper we propose an efficient and effective LSF-FR scheme, namely monogenic binary coding (MBC), which encodes the local pattern in different monogenic feature maps. The BFLD [22] is then adopted to extract the low-dimensional discriminative features from the generated MBC feature maps of amplitude, phase and orientation, respectively. Finally, the three types of LSFs are fused for face classification. One of the most important advantages of the proposed MBC scheme is its low time and space complexity while achieving very competitive performance with the Gaborfeature based LSF-FR methods [9][10][11][22]. The proposed MBC method is validated on benchmark large scale face databases, including Multi-PIE [49], FERET [14][41], FRGC 2.0 [13] and PolyU-NIR [28]. The experimental results verified the efficiency and effectiveness of the proposed MBC based LSF-FR method.

The rest of the paper is organized as follows. Section 2 introduces briefly the monogenic signal representation. Section 3 presents in detail the MBC algorithm. Section 4 describes the whole scheme of MBC based LSF-FR. Section 5 presents the experimental results, and Section 6 concludes the paper. 


\section{Monogenic Signal Representation}

Monogenic signal is an important generalization of the analytic signal from one dimension (1D) to two dimensions (2D), and it preserves the desired properties of 1D analytic signal. One prominent property of monogenic signal representation lies in that its feature extraction process (phase, amplitude and orientation estimation) is truly rotation-invariant [32]. Monogenic signal representation has been used in many applications such as face recognition [23], scale-space analysis [31], and texture classification [33]. In this section, we first briefly introduce the analytic signal, and then describe the monogenic signal representation.

\subsection{Analytic signal representation}

The analytic signal is a complex-valued representation in 1D signal processing. Given a real valued 1D signal $f(x)$, its complex analytic signal is defined as

$$
f_{\text {anal }}(x)=f(x)+j \cdot f_{\mathrm{H}}(x)
$$

where $f_{\mathrm{H}}(x)=f(x) * h(x)$, and $h(x)=1 / \pi x$ refers to the Hilbert transform kernel in the spatial domain. In general the Hilbert transform is performed in frequency domain and the response of $h(x)$ in frequency domain is $H(\omega)=-j \cdot \operatorname{sign}(\omega)=-j \omega /|\omega| \cdot$

Table 1: Some 1D features and their corresponding phase angles (degree).

\begin{tabular}{|c|c|c|c|c|c|c|}
\hline Feature & $\subsetneq$ & $\neg$ & $\searrow$ & $\wedge$ & $\Gamma$ & $\searrow$ \\
\hline $\begin{array}{c}\text { Feature } \\
\text { type }\end{array}$ & $\begin{array}{c}\text { step edge } \\
\text { (Positive going) }\end{array}$ & $\begin{array}{c}\text { Step edge } \\
\text { (Negative going) }\end{array}$ & $\begin{array}{c}\text { Spot } \\
\text { (Negative going) }\end{array}$ & $\begin{array}{c}\text { Spot } \\
\text { (Positive going) }\end{array}$ & $\begin{array}{c}\text { Roof edge } \\
\text { (Positive) }\end{array}$ & $\begin{array}{c}\text { Roof edge } \\
\text { (Negative) }\end{array}$ \\
\hline $\begin{array}{c}\text { Phase } \\
\text { angle }\end{array}$ & 0 & 180 & 270 & 90 & 44.2 & 224.2 \\
\hline
\end{tabular}

The analytic signal representation allows one to retrieve the local amplitude $A(x)$ and local phase $\varphi(x)$ :

$$
\begin{gathered}
A(x)=\left\|f_{\text {anal }}(x)\right\|=\sqrt{f^{2}(x)+f_{\mathrm{H}}^{2}(x)} \\
\varphi(x)=\operatorname{atan2}\left(f_{\mathrm{H}}(x), f(x)\right), \varphi(x) \in[0,2 \pi)
\end{gathered}
$$

where the local phase $\varphi(x)$ is invariant with respect to the local energy of the signal but changes if the local structure varies, and the local amplitude $A(x)$ is invariant with respect to the local structure but represents the local energy. Table 1 shows the relation between feature type and phase angle [18], respectively. 


\subsection{Monogenic signal representation}

Monogenic signal was introduced by Felsberg and Sommer in 2001 [12] to generalize the analytic signal from 1D to 2D. The monogenic signal is built around the Riesz transform which is a natural multidimensional extension of the Hilbert transform [19][32]. The Riesz transform is the scalar-to-vector signal transformation whose frequency response is $-j \omega /\|\omega\|$. In 2D, the Riesz transform can be expressed as

$$
\boldsymbol{f}_{\mathrm{R}}(\boldsymbol{z})=\left(\begin{array}{l}
f_{x}(\boldsymbol{z}) \\
f_{y}(\boldsymbol{z})
\end{array}\right)=\left(\begin{array}{l}
h_{x} * f(\boldsymbol{z}) \\
h_{y} * f(\boldsymbol{z})
\end{array}\right)
$$

where $f(z)$ with $z=(x, y)$ is the input signal, and filters $h_{x}$ and $h_{y}$ are characterized by the 2D frequency responses $H_{x}=-j \omega_{x} /\|\boldsymbol{\omega}\|$ and $H_{y}=-j \omega_{y} /\|\boldsymbol{\omega}\|$ with $\boldsymbol{\omega}=\left(\omega_{x}, \omega_{y}\right)$. It is easy to obtain the spatial representation of Riesz kernel, which is

$$
\left(h_{x}, h_{y}\right)=\left(\frac{x}{2 \pi\|z\|^{3}}, \frac{y}{2 \pi\|z\|^{3}}\right)
$$

For an image $f(z)$, its monogenic signal is defined as the combination of $f$ and its Riesz transform

$$
f_{\mathrm{M}}(z)=\left(f(z), f_{x}(z), f_{y}(z)\right)
$$

In Eq. (6), $f(z)$ is called the real part of the monogenic signal, and $f_{x}(z)$ and $f_{y}(z)$ the two imagery parts. Based on the real and imagery parts, the original image signal $f(z)$ could be orthogonally decomposed into three components, local amplitude, local phase and local orientation, which are defined as [12]

$$
\left\{\begin{array}{l}
\text { local amplitude: } A=\sqrt{f^{2}+f_{x}^{2}+f_{y}^{2}} \\
\text { local phase: } \phi=-\operatorname{sign}\left(f_{x}\right) \operatorname{atan} 2\left(\sqrt{f_{y}^{2}+f_{x}^{2}} / f\right) \\
\text { local orientation: } \theta=\operatorname{atan}\left(f_{y} / f_{x}\right)
\end{array}\right.
$$

where $A$ describes the local energetic information, $\phi$ describes the local structural information, and $\theta$ describes the geometric information.

The local phase and local orientation compose the monogenic phase of a 2D signal [12]. In contrast to 1D case, the monogenic phase includes additional geometric information, which indicates the main orientation in ideal case (i.e., the signal is constant in one direction). Two toy examples of image features (e.g., step and roof) are shown in Fig. 2. Fig. 2(a) shows the step edge and roof edge features in 2D image, with Fig. 2(b) the corresponding 3D shapes of them. The geometric information (i.e., the main orientation) is illustrated by local orientation shown in Fig. 2(a), with the structure information shown by local phase in Fig. 2(c). 

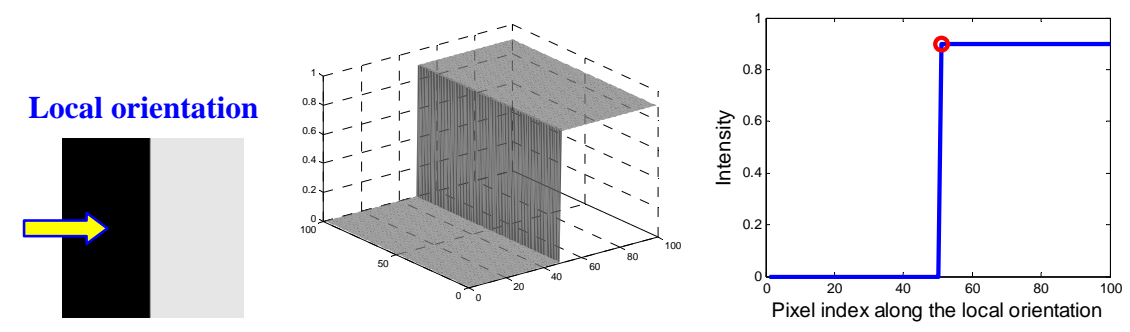

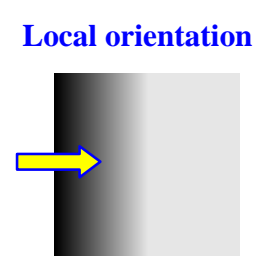

(a)

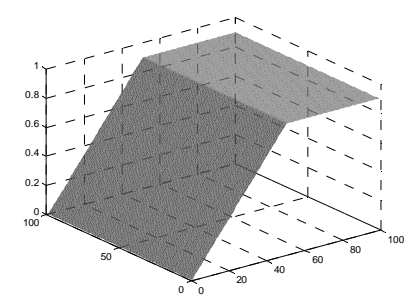

(b)

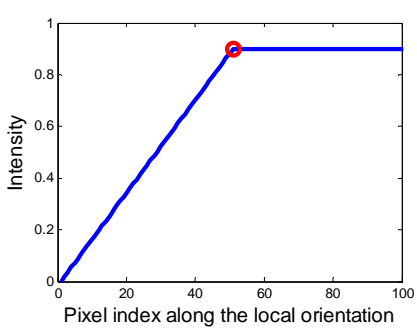

(c)

Figure 2: Toy examples of monogenic phase. (a) Two image structures; (b) their shapes shown in 3D; and (c) the monogenic phases of them.

\subsection{Multi-resolution monogenic signal representation}

In practice, the signals are of finite length, and we need to perform band-pass filtering to an image before applying the Riesz transforms. On the other side, as indicated by [12], band-pass filtering has the benefits of maintaining the invariance-equivariance property of signal decomposition. Here the invariance-equivariance property [21][12] means that energy (local amplitude) and structure (local phase on local orientation) are independent information.

Classical Gabor filters are commonly used as band-pass filters for that they could offer the best simultaneous localization of spatial and frequency. However, these filters overlap more in low frequencies than in high frequencies, leading to a non-uniform coverage of the Fourier domain. Moreover, Gabor filters have non-zero means, and thus they are affected by direct current (DC) components [34]. An alternative to the Gabor filters is the log-Gabor filters proposed in [20]. The log-Gabor filters discard the DC components and can overcome the bandwidth limitation of traditional Gabor filters. Meanwhile, it has a Gaussian shaped response along the logarithmic frequency scale instead of a linear one. This allows more information to be captured in the high frequency band and endows desirable high pass characteristics.

The frequency response of log-Gabor filters can be described as

$$
G(\omega)=\exp \left\{-\left[\log \left(\omega / \omega_{0}\right)\right]^{2} / 2\left[\log \left(\sigma / \omega_{0}\right)\right]^{2}\right\}
$$

where $\omega_{0}$ is the center frequency and $\sigma$ is the scaling factor of the bandwidth. In order for filters with the 
constant shape ratio, we set $\sigma / \omega_{0}$ as a constant as suggested in [43]. It is worth noting that a log-Gabor filter with a 3-octave bandwidth has the same spatial width as that of a 1-octave Gabor filter, demonstrating its ability to capture broad spectral information with compact support [42].

The band-pass monogenic signal representation is defined as

$$
f_{\mathrm{lg}-\mathrm{M}}(z)=\left(f_{\mathrm{lg}}(z), f_{\mathrm{lg}-x}(z), f_{\mathrm{lg}-y}(z)\right)=\left(f_{\mathrm{lg}}(z), h_{x} * f_{\mathrm{lg}}(z), h_{y} * f_{\mathrm{lg}}(z)\right)
$$

where $f_{\mathrm{lg}}(\boldsymbol{z})=f(z) * F^{-1}(G(\omega))$, and $F^{-1}$ represents the 2D inverse Fourier transform. Similarly, the local amplitude $A_{\mathrm{lg}}$, orientation $\theta_{\mathrm{lg}}$ and phase $\phi_{\mathrm{gg}}$ of a 2D signal $f$ can be computed by [12]

$$
A_{\mathrm{lg}}=\sqrt{f_{\mathrm{lg}}^{2}+f_{\mathrm{lg}-x}^{2}+f_{\mathrm{lg}-y}^{2}} ; \theta_{\mathrm{lg}}=\operatorname{atan}\left(f_{\mathrm{lg}-y} / f_{\mathrm{lg}-x}\right) ; \phi_{\mathrm{lg}}=-\operatorname{sign}\left(f_{\mathrm{lg}-x}\right) \operatorname{atan} 2\left(\sqrt{f_{\mathrm{lg}-y}^{2}+f_{\mathrm{lg}-x}^{2}} / f_{\mathrm{lg}}\right)
$$

Since log-Gabor filters are band-pass filters, usually multi-scale monogenic representation is required to fully describe a signal. In multi-scale log-Gabor filters, the parameters $\sigma$ and $\omega_{0}$ can be rewritten as

$$
\sigma=\sigma_{\text {ratio }} \omega_{0}, \omega_{0}=\left(\lambda_{\min } \mu^{s-1}\right)^{-1}
$$

where $\lambda_{\min }$ is the minimal wavelength, $\mu$ is the multiply factor of wavelength, $s$ is the scale index, and $\sigma_{\text {ratio }}$ is the ratio $\sigma / \omega_{0}$.

As an example, Fig. 3 shows the monogenic representation of a face image on one scale. We can see that the amplitude components reflect the local variation of gray value; while the facial local structures are well captured in the local phase and orientation components.

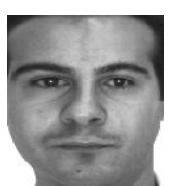

(a)

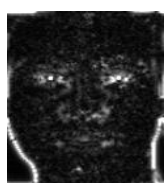

(b)

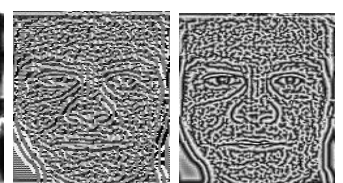

(d)

Figure 3: (a) A face image and its monogenic representation on one scale: (b) amplitude component, (c) orientation component and (d) phase component.

\subsection{Multi-scale monogenic signal representation vs. multi-scale Gabor wavelet representation}

Traditional 2D Gabor filters are highly jointly localized in spatial location, orientation and frequency. Usually 5-scale and 8-orientation Gabor filters are used in face recognition [7][9][10][11][22][36], where the Gabor features at each orientation are extracted by using steerable filters. 
It is easy to see that both multi-scale 2D Gabor wavelet (i.e., Gabor filters) representation and multi-scale monogenic signal representation are redundant. For multi-scale Gabor wavelets, the redundancy comes from two aspects: one is the redundancy across multiple scales, and the other is the redundancy across multiple orientations on each scale. However, for multi-scale monogenic signal representation, the redundancy only comes from the multi-scale representation, because on each scale the local amplitude, local phase, and local orientation are orthogonal. Although multi-scale Gabor filtering is effective for FR, the high cost of Gabor representation (80 convolutions for complex Gabor filtering across 5 scales and along 8 orientations) limits its application in practice. On the other side, although the multi-scale monogenic signal representation has no redundancy along orientation, this does not mean that it is not effective for face representation. Our previous work in [23] has shown that the combination of orientation and amplitude components of monogenic signal representation could achieve even better recognition rates than the multi-scale Gabor wavelet representation but with significantly lower time and space complexity.

\section{Monogenic Binary Coding}

In this section, we describe the proposed algorithm of coding the monogenic signal features, which contains two parts: monogenic local variation coding and monogenic local intensity coding. The first part encodes the variation between the central pixel and its surrounding pixels in a local patch, while the second part encodes the value of central pixel itself. Obviously, these two parts are complementary.

\subsection{Binary coding of monogenic local variation}

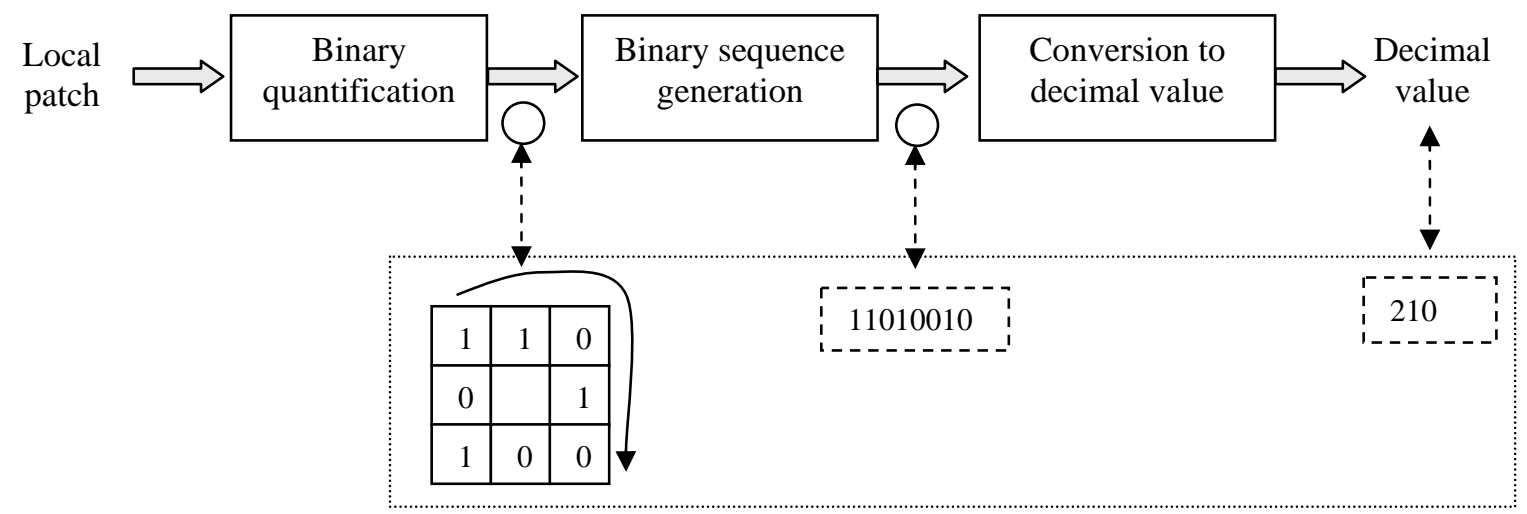

Figure 4: The typical process of local variation coding. 
Refer to Fig. 4, for each local patch (e.g., 3×3 neighborhood) a typical local variation coding usually consists of the following three stages: binary quantification, binary sequence generation, and binary sequence to decimal value conversion. In the procedures of local variation coding, the binary sequence generation and binary sequence to decimal value conversion are the common stages in most binary pattern coding methods, such as LBP [8][16], LGBP [9], HGPP [11] and LGXP [22]. An example of these two steps is shown in the second row of Fig. 4. The decimal value of the binary code is defined as follows:

$$
C\left(\boldsymbol{z}_{c}\right)=\left[C_{N}, C_{N-1}, \ldots, C_{1}\right]_{\text {binary }}=\left[\sum_{i=1}^{N} 2^{i-1} \cdot C_{i}\right]_{\text {decimal }}
$$

where $\boldsymbol{z}_{c}$ denotes the central pixel position in the local patch of feature map, $C_{i}$ denotes the binary code ( 0 or 1) of the $i^{\text {th }}$ neighbor of the central pixel and $N$ is the number of involved neighbors (we fix $N=8$ ).

However, the first step of local variation coding, i.e., binary quantization, usually depends on the property of feature map, and should be designed based on the physical meaning of the feature. For example, in the binary quantization of LBP [8] which regards original image as feature map, the surrounding pixel is coded as 1 if its value is not less than the value of central pixel; otherwise, the surrounding pixel is coded to 0 . In HGPP [11], the surrounding pixel is coded to 0 if it has the same sign as that of the central pixel; otherwise, it is quantified to 1 . The monogenic signal representation has three components: local amplitude, local orientation and local phase. The local orientation and local phase compose monogenic phase. According to the properties of local amplitude and monogenic phase, different binary quantization strategies are utilized to generate the binary code.

a) Local variation of monogenic amplitude: The local amplitude of monogenic signal representation is a measurement of local energetic information. For example, high amplitude usually indicates higher energetic local features (e.g., edges, lines, textures). Therefore, the local variation of monogenic amplitude could be coded by comparing the amplitude value of central location with those of its neighbors, which is similar to LBP [8]. For a local patch, let's denote by $A\left(\boldsymbol{z}_{c}\right)$ the amplitude value of the central pixel $\boldsymbol{z}_{c}$, and by $A(i)$ the amplitude value of the $i^{\text {th }}$ neighbor of the central pixel. Usually, we normalize the range of the amplitude to $[0,255]$. Then the amplitude binary code of the $i^{\text {th }}$ neighbor is defined as

$$
C_{i}^{A}\left(\boldsymbol{z}_{c}\right)= \begin{cases}1 & \text { if } A(i) \geq A\left(\boldsymbol{z}_{c}\right) \\ 0 & \text { else }\end{cases}
$$


Consequently, the amplitude binary code, denoted by $C_{A}$, of the central pixel $\boldsymbol{z}_{c}$ is formed as $C_{A}\left(\boldsymbol{z}_{c}\right)=\left[C_{N}^{A}\right.$, $\left.C_{N-1}^{A}, \ldots, C_{1}^{A}\right]_{\text {binary }}$.

b) Local variation of monogenic phase: For 2D signals, monogenic phase is decomposed into local orientation and local phase. The local orientation describes the geometric information, more specifically the main orientation of the local structure, and the local phase describes the information of the local structure along the main orientation (i.e., structure type as shown in Table 1). Both local orientation and local phase are represented by angle degree. In this paper, we unwrap both of them into the range of [0,360). Because the angle of local phase or local orientation indicates the structural or geometric information, it is more meaningful to compare the feature type than to directly compare the angle's degree. Therefore, the binary coding of local phase and local orientation variations should adopt a different strategy from that of local amplitude. Inspired by the coding strategy of Gabor phase in HGPP [11] and LGXP [22], we consider two cases when comparing the monogenic features: one is that the local phases/orientations are the same or close enough to each other and the other is that the local phases/orientations are different.

In order to increase the robustness of binary coding, we firstly divide the range $[0,360)$ into $K$ intervals (we fix $K=4$ in this paper). If two phases/orientations belong to the same interval, they are believed to be similar local features; otherwise, they are regarded as different. For a local patch, denote by $\Phi\left(\boldsymbol{z}_{c}\right)$ the local phase of the central pixel $\boldsymbol{z}_{c}$, and by $\Phi(i)$ the phase of its $i^{\text {th }}$ neighbor. The phase binary code of the $i^{\text {th }}$ neighbor is

$$
C_{i}^{P}\left(\boldsymbol{z}_{c}\right)= \begin{cases}0 & \text { if } Q\left(\Phi\left(\boldsymbol{z}_{c}\right)\right)=Q(\Phi(i)) \\ 1 & \text { else }\end{cases}
$$

where $Q(x)$ is the quantification function, defined as

$$
Q(x)=q \quad \text { if } \frac{360 \cdot(q-1)}{K} \leq x<\frac{360 \cdot q}{K}
$$

Then the local phase binary code, denoted by $C_{P}$, of the central pixel $\boldsymbol{z}_{c}$ is formed as $C_{P}\left(\boldsymbol{z}_{c}\right)=\left[\mathrm{C}_{N}^{P}, \mathrm{C}_{N-1}^{P}, \ldots\right.$, $\left.C_{1}^{P}\right]_{\text {binary }}$

Similarly, let's denote by $O\left(\boldsymbol{z}_{c}\right)$ the local orientation of the central pixel $\boldsymbol{z}_{c}$, and by $O(i)$ the local orientation of its $i^{\text {th }}$ neighbor. The orientation binary code of the $i^{\text {th }}$ neighbor is

$$
C_{i}^{O}= \begin{cases}0 & \text { if } Q\left(O\left(z_{c}\right)\right)=Q(O(i)) \\ 1 & \text { else }\end{cases}
$$


And then the local orientation binary code, denoted by $C_{O}$, of the central pixel $\boldsymbol{z}_{c}$ is formed as $C_{O}\left(\boldsymbol{z}_{c}\right)=\left[C_{N}^{O}\right.$, $\left.C_{N-1}^{o}, \ldots, C_{1}^{O}\right]_{\text {binary }}$

\subsection{Binary coding of monogenic local imagery intensity}

Almost all the LSF-FR methods, including LBP [8], LGBP [9] and LGXP [22], code the variation information of a patch for pattern recognition but omit the feature information of the central pixel in the local patch. In fact, the information of central pixel itself can have discriminative information which may not be carried out by the local variation. For instance, the two pixels having the same local variation pattern may have very different intensities. In [25], Guo et al. proposed to quantify the local intensity of central pixel based on the average gray level of the whole image for texture classification; however, this binary quantization scheme may not be robust, especially for FR with illumination changes.

In the monogenic signal representation, the Riesz transform is asymmetric and it can suppress the DC component [12]. Such properties make the imaginary part of Riesz transform able to enhance the feature intensity while being robust to disturbances such as illumination changes. Therefore, in this paper we propose to use the imagery part of monogenic signal representation to encode the local feature intensity information of the central pixel. Since there are two components (e.g., $f_{\mathrm{lg}-x}$ and $f_{\mathrm{lg}-y}$ ) in the imagery part of monogenic signal representation, the binary coding leads to a quadrant bit for the monogenic imagery feature at a location, as illustrated in Fig. 5.

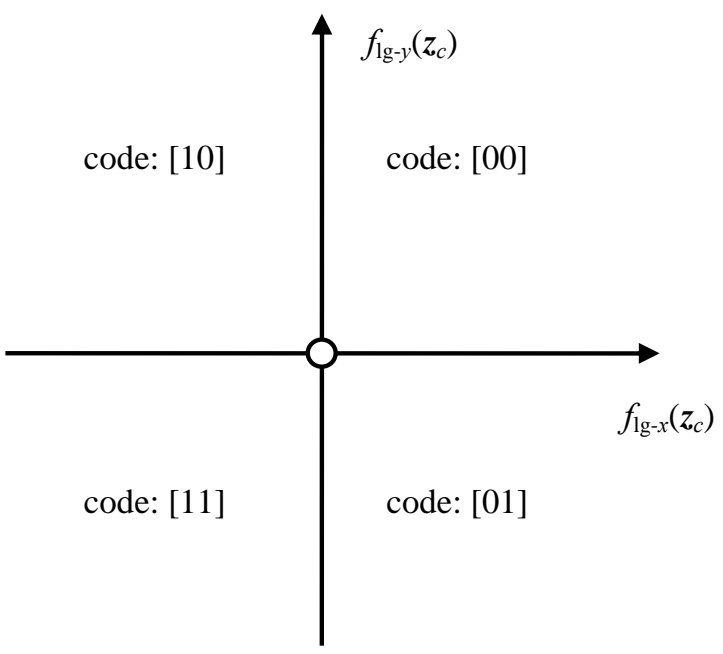

Figure 5: Quadrant bit coding of monogenic imaginary feature at each location. 
Refer to Fig. 5, the imagery part of monogenic signal representation at pixel $\boldsymbol{z}_{c}$ is encoded into two bits, $\left[C_{x}^{l}\left(\boldsymbol{z}_{c}\right), C_{y}^{l}\left(\boldsymbol{z}_{c}\right)\right]_{\text {binary }}$, by the following rule:

$$
C_{d}^{I}\left(\boldsymbol{z}_{c}\right)=\left\{\begin{array}{ll}
0 & \text { if } f_{\mathrm{lg}-d}\left(\boldsymbol{z}_{c}\right)>0 \\
1 & \text { if } f_{\mathrm{lg}-d}\left(\boldsymbol{z}_{c}\right) \leq 0
\end{array}, \quad d \in\{x, y\}\right.
$$

where $f_{\mathrm{lg}-x}$ and $f_{\mathrm{lg}-y}$ (refer to Eq. (9) please) are respectively the horizontal and vertical Riesz transform outputs of monogenic signal representation.

\subsection{Monogenic binary code (MBC)}

With the coding procedures described in Sections 3.1 and 3.2, for each of the amplitude, phase and orientation components of the monogenic signal representation, we can have a monogenic binary code (MBC) map. We denote by MBC-A, MBC-P and MBC-O the code maps for amplitude, phase and orientation, respectively. At each location, MBC-A, MBC-P and MBC-O are formed by combining the local imagery intensity code and the local variation code as follows

$$
\begin{aligned}
& \operatorname{MBC}-\mathrm{A}\left(\boldsymbol{z}_{c}\right)=\left[C_{x}^{l}\left(\boldsymbol{z}_{c}\right), C_{y}^{l}\left(\boldsymbol{z}_{c}\right), C_{A}\left(\boldsymbol{z}_{c}\right)\right]_{\text {binary }} \\
& \operatorname{MBC-P}\left(\boldsymbol{z}_{c}\right)=\left[C_{x}^{l}\left(\boldsymbol{z}_{c}\right), C_{y}^{l}\left(\boldsymbol{z}_{c}\right), C_{P}\left(\boldsymbol{z}_{c}\right)\right]_{\text {binary }} \\
& \operatorname{MBC-O}\left(\boldsymbol{z}_{c}\right)=\left[C_{x}^{l}\left(\boldsymbol{z}_{c}\right), C_{y}^{l}\left(\boldsymbol{z}_{c}\right), C_{O}\left(\boldsymbol{z}_{c}\right)\right]_{\text {binary }}
\end{aligned}
$$

Table 2: The numbers of patterns in MBC-X $(X \in\{A, P, O\}), L B P$, and Gabor-related coding methods (e.g., LGBP, HGPP and LGXP) when the 8 closest neighbors of a pixel are involved in coding.

\begin{tabular}{llll}
\hline Method & MBC-X & LBP & Gabor-related coding methods \\
\hline Number of patterns & 1024 & 256 & 256 \\
\hline
\end{tabular}

It can be seen that when the 8 closest neighbors of a pixel are involved in local variation coding, each MBC pattern will have 10 bits. Then the number of possible patterns for each MBC is 1024, which is larger than that of previous binary coding methods such as LBP, LGBP, HGPP and LGXP (refer to Table 2 please). The more patterns can characterize more accurately the local signal structure; at the same time, it will also make the generated histogram in each sub-region a little sparser. However, this sparser histogram can still have enough (even higher) discrimination capability and result in very good FR performance (please refer to the section of experimental results). 
The above three MBC maps can be used individually as the feature for face classification, and they can also be used together to enhance the FR performance, as we will describe in the next section.

\section{Face Recognition by Monogenic Binary Code}

\subsection{Face recognition by individual MBC}

The statistical information of image local areas can be described by local histograms, which are robust to the image occlusion and variations of pose, expression, and noise, etc. After computing the multi-scale MBC-A, MBC-P and MBC-O feature maps of the face image, we can construct three histograms, $H_{\mathrm{MBC}-\mathrm{A}}, H_{\mathrm{MBC}-\mathrm{P}}$ and $H_{\mathrm{MBC}-\mathrm{o}}$, through the following procedures. For each kind of pattern map on each scale, it is partitioned into multiple non-overlapping regions, and then the local histogram is built for each sub-region. Finally, all the local histograms across different scales and different regions are concatenated into a single histogram vector to represent the face image. Formally, the $H_{\mathrm{MBC}-\mathrm{A}}, H_{\mathrm{MBC}-\mathrm{P}}$ and $H_{\mathrm{MBC}-\mathrm{O}}$ histograms are formulated as

$$
H_{t}=\left(h_{t}(r, s), r=1, \cdots, L ; s=1,2,3\right), \quad t \in\{\text { MBC-A, MBC-P, MBC-O }\}
$$

where $L$ is the number of sub-regions on each scale; $s$ denotes the scale index; and $h_{t}(r, s)$ is the histogram of feature map $t$ in the $r^{\text {th }}$ sub-region on scale $s$.

To be identical to other LSF based FR methods [9-11][22], the similarity between two histograms $h_{a}$ and $h_{b}$ is defined as their intersection:

$$
S_{H}\left(h_{a}, h_{b}\right)=\sum_{i=1}^{M} \min \left(h_{a}^{i}, h_{b}^{i}\right)
$$

where $M$ is the number of bins in the histogram, and $h_{a}^{i}$ and $h_{b}^{i}$ denote the frequency of the $i^{\text {th }}$ bin in $h_{a}$ and $h_{b}$, respectively. By measuring the histogram intersection, the similarity of $H_{\mathrm{MBC}-\mathrm{A}}, H_{\mathrm{MBC}-\mathrm{P}}$ and $H_{\mathrm{MBC}-\mathrm{O}}$ histogram features from gallery and probe face images could be computed for classification.

\subsection{Face recognition by fused MBC}

It is obvious that different sub-regions in the face image will have different discriminative power for FR. Therefore, many methods [8-11][23] assign different weights to the histograms of different sub-regions before matching. This is actually a feature combination process and could improve the FR rate. However, this weighting scheme cannot reduce the feature dimensionality. Considering that the kernel linear 
discrimination analysis adopted in [26][36] has high time complexity, we adopt the BFLD [22] scheme to reduce the histogram feature dimension while enhancing its discrimination. The MBC feature map is first partitioned into $M_{b} \times M_{b}$ blocks, and then each block is further partitioned into $M_{r} \times M_{r}$ sub-regions. In each sub-region, the histogram of feature map on each scale is built as described in Section 4.1. The histogram feature of each block is the concatenation of all the histograms of its sub-regions. Then for the histogram feature of each block, LDA [6] is used to learn a projection matrix from the training set, and then the dimensionality reduced histogram feature can be obtained by using this projection matrix.

Denote by $F_{t}^{p}(i)$ and $F_{t}^{g}(i)$ the dimensionality reduced features of $H_{t}$ in the $i^{\text {th }}$ block of a probe face image and a gallery image, respectively, where $t \in\{$ MBC-A, MBC-P, MBC-O $\}$. Since $F_{t}^{p}$ and $F_{t}^{g}$ are not histogram features anymore, we compute their similarity using the cosine distance:

$$
S_{t}\left(F_{t}^{p}(i), F_{t}^{g}(i)\right)=\frac{\left\langle F_{t}^{p}(i), F_{t}^{g}(i)\right\rangle}{\left\|F_{t}^{p}(i)\right\|\left\|F_{t}^{g}(i)\right\|}
$$

where $\langle\bullet\rangle$ is the inner product operator. The cosine distance is widely used in face recognition [22][26][36], and in [45] it has been reported that the cosine distance performs better than $L_{1}, L_{2}$ and Mahalanobis distances in most face recognition experiments. In addition, using cosine distance could also make the comparison with [22] fair. The similarity between the whole probe and gallery images is computed as

$$
S_{t}\left(F_{t}^{p}, F_{t}^{g}\right)=\sum_{i=1}^{M_{b} \times M_{b}} S_{t}\left(F_{t}^{p}(i), F_{t}^{g}(i)\right)
$$

The monogenic amplitude, phase and orientation components describe the energetic, structural and geometric information of a 2D signal, respectively, and they are complementary to each other. Therefore, it is useful to fuse these three types of features for a more robust FR result. In this paper, we use the simple weighted average to fuse the three similarities, i.e., $S_{\mathrm{MBC}-\mathrm{A}}, S_{\mathrm{MBC}-\mathrm{P}}$ and $S_{\mathrm{MBC}-\mathrm{o}}$, as follows

$$
S_{\mathrm{MBC}-\mathrm{F}}=(1-2 \cdot w) S_{\mathrm{MBC}-\mathrm{A}}+w S_{\mathrm{MBC}-\mathrm{P}}+w S_{\mathrm{MBC}-\mathrm{O}}
$$

where $w$ is the weight. Note that in Eq. (25) we let the weights assigned to $S_{\mathrm{MBC}-\mathrm{P}}$ and $S_{\mathrm{MBC}-\mathrm{O}}$ equal because the local orientation and local phase have almost the same performance in various databases. Therefore, there is only one weight parameter, i.e., $w$, to be determined in our fusion scheme, and accordingly we call the FR scheme with similarity score $S_{\mathrm{MBC}-\mathrm{F}}$ as fused MBC, denoted by MBC-F. 


\section{Experimental Results}

In this section, we first discuss the parameter settings, and then give the time and space complexity comparison between MBC and some representative and state-of-the-art LSF-FR methods, including LGBP [9], HGPP [11] and LGXP [22]. Finally, we evaluate the proposed MBC algorithm on four large scale benchmark face databases: Multi-PIE [49], FERET [14][41], FRGC 2.0 [13] and PolyU-NIR[28]. The Matlab source code of the proposed MBC algorithm can be downloaded at http://www4.comp.polyu.edu.hk/ cslzhang/code.htm.

\subsection{Parameter settings}

There are some parameters in different stages of MBC, e.g., multi-scale log-Gabor filtering, sub-region histogram computing, and feature combination by BFLD [22]. In order to be consistent with other methods and provide a fair comparison, no pre-processing is used in all the following experiments.

Refer to Section 2.3, the parameters in multi-scale log-Gabor filtering are the minimal wavelength $\lambda_{\text {min }}$, the ratio factor $\sigma_{\text {ratio }}$, the multiply factor of wavelength $\mu$ and the number of scale (i.e., the scale index $s$ ). In our paper, if no specific instruction, we fix $\lambda_{\min }=4, \mu=0.64, \sigma_{\text {ratio }}=1.7$, and the number of scale as 3 . Because one-scale log-Gabor filter could capture more frequency information than one-scale Gabor filter [42][43], the number of scales in multi-scale log-Gabor filtering is often set to 3 [23][47][48]. Since the number of scales will affect much the time and space complexity of the proposed method, we perform two experiments on Fb and DupI datasets of the FERET database to discuss the selection of scale parameter $s$.

The detailed experimental settings on Fb and DupI are presented in Section 5.4. We change the number of scales from 1 to 4, and plot the FR rates of MBC versus the scale parameter $s$ in Fig 6 . It could be seen that in most cases (especially for the test in DupI), the recognition rate will benefit from the increase of $s$ from 1 to 3 or 4 . It is interesting to see that in the test on $\mathrm{Fb}$, there are some fluctuations as the scale number increases. This is possibly because the test data in Fb has little variation compared to the gallery set Fa, so it is not necessary to use multi-scale representation. Nevertheless, considering the balance of recognition rate and complexity, particularly in more challenging FR scenarios (e.g., DupI), we prefer to set the scale number to 3 in all the experiments. 


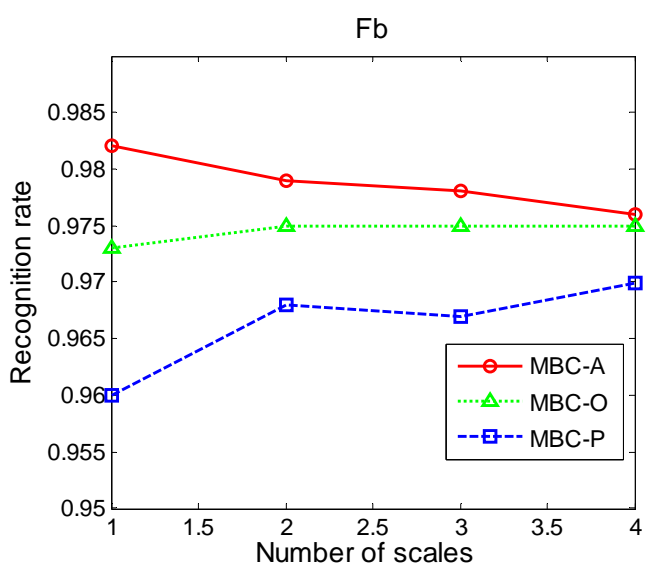

(a)

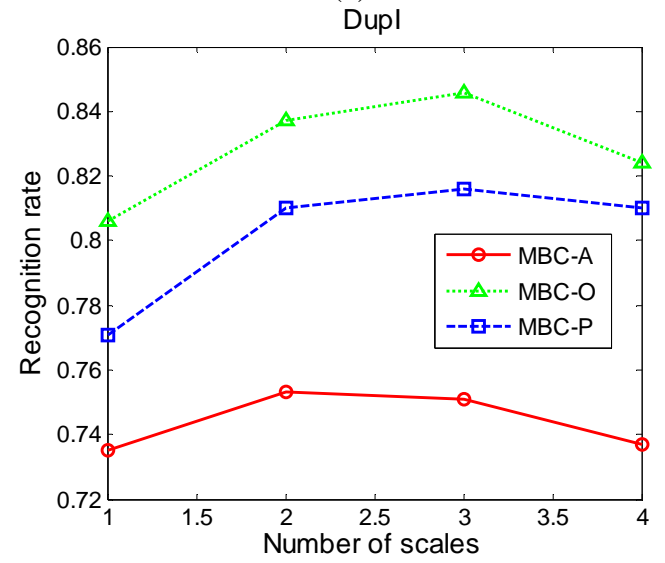

(c)

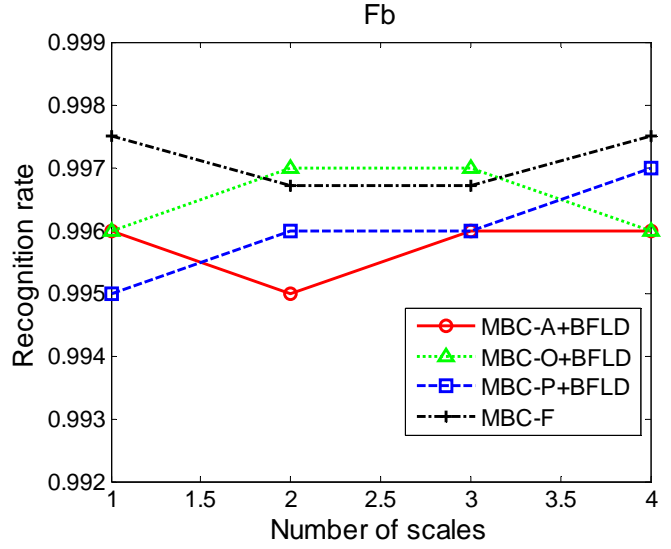

(b)

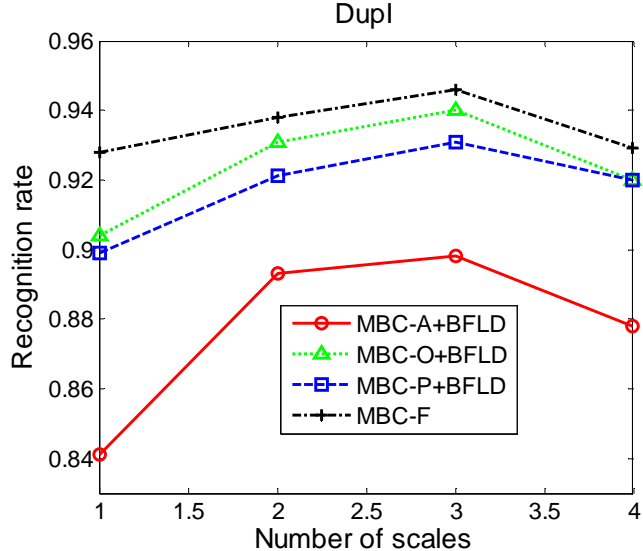

(d)

Figure 6: The curves of FR rate versus the number of scales by the proposed MBC methods. (a) MBC on Fb; (b) MBC+BFLD and MBC-F on Fb; (c) MBC on DupI; (d) MBC+BFLD and MBC-F on DupI.

In the stage of sub-region histogram computing, the whole image needs to be divided into $M_{b} \times M_{b}$ blocks, which is further divided into $M_{r} \times M_{r}$ sub-regions. In each sub-region, the histogram is extracted; and in each block, the discriminative feature is extracted by BFLD. In the stage of fusing, three similarities, i.e., $S_{\mathrm{MBC}-\mathrm{A}}$ $S_{\mathrm{MBC}-\mathrm{P}}$ and $S_{\mathrm{MBC}-\mathrm{O}}$, will be averaged by using the weight $w$. If no specific instruction, we fix $M_{r}=2, M_{b}=4, w$ $=0.275$, and the dimensionality of discriminative feature in each block as 200 .

\subsection{Time and space complexities}

In the representative LSF based FR methods [8][9][10][11][22][23], the feature map generation, pattern map coding, histogram computing, and histogram similarity computing are the main time-consuming parts. The number of image convolution directly decides the computational burden of feature map generation, while the number of pattern maps directly affects the running time of other parts and space complexity of algorithms. Fig. 7 compares the time and space complexity of MBC (including MBC-A, MBC-O and MBC-P) and 
MBC-F with some state-of-the-art LSF-FR methods, including LGBP [9], HGPP [11], LGXP [22], and LGB\&XP (fusing Gabor magnitude and phase parts in [22]).

All the methods of LGBP, HGPP, LGXP, and LGB\&XP use Gabor transformations (with 5 scales and 8 orientations) to generate feature maps, which means that 80 convolutions (40 real convolutions and 40 imagery convolutions) are needed in processing a single image. However, for the process of feature generation in the proposed MBC and MBC-F, only 9 convolutions are executed (1 log-Gabor filtering and 2 Riesz transformations on each scale) [12][23][32][33]. Fig. 7 clearly shows that the time complexity of MBC in feature map generation is only 1/9 times of that of [9][10][11][22].

In the pattern map coding, both LGBP and LGXP encode 40 feature maps into 40 pattern maps, and HGPP produces 90 pattern maps, including 10 global phase pattern maps and 80 local pattern maps, with LGB\&XP outputting 40 Gabor magnitude and 40 Gabor phase pattern maps. In comparison, our proposed MBC-A, MBC-P and MBC-O each only needs to encode 3 monogenic pattern maps. Even the fusing version of MBC, i.e., MBC-F, only has 9 pattern maps together. Therefore the proposed MBC has significantly lower time and space complexity than the previous Gabor-based ones [9][10][11][22] in pattern map coding, as illustrated in Fig. 7.

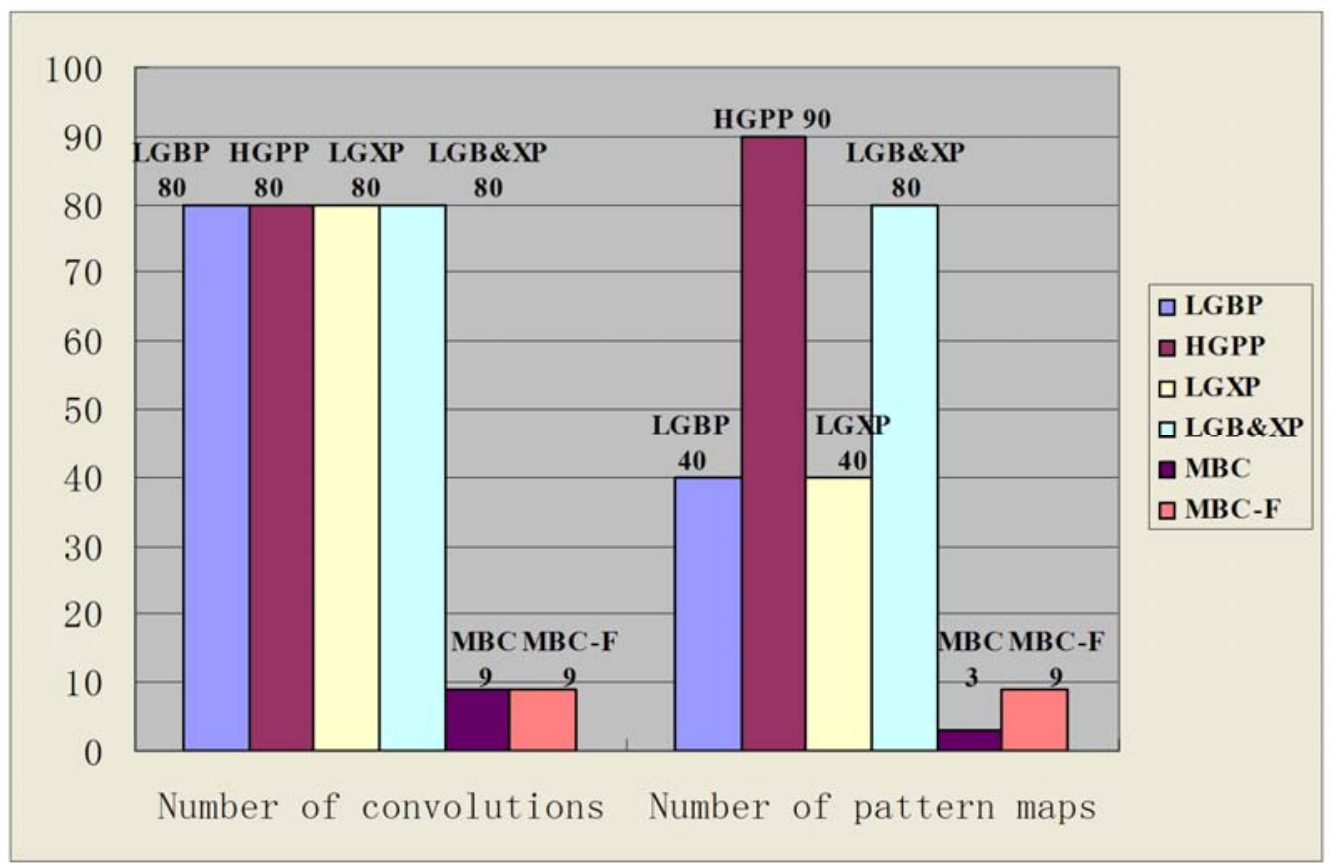

Figure 7: Comparison of the time and space complexities among LGBP [9], HGPP [11], LGXP [22], LGB\&XP[22] (fusing LGXP and LGBP) and the proposed MBC (MBC denotes any one of MBC-A, MBC-P, and MBC-O) and MBC-F. 
Considering that the time and space complexity for histogram generating depends on the number of pattern maps, the proposed MBC methods will also need much less computational cost and space in histogram feature generation than [9][10][11][22].

In similarity computing, when the original histogram features are used, according to the number of pattern maps, the time and space complexity of proposed MBC scheme is much less than that of LGBP, HGPP and LGXP. However, when BFLD [22] is used to reduce the dimensionality of histogram features, the dimensionality of projected discriminative features for all methods, including MBC and LGBP[9], HGPP[11], LGXP[22], is nearly the same as each other. Therefore, when coupled with BFLD [22], all the methods would have similar time and space complexity in the phase of similarity computing. Note that BFLD will increase the computational complexity in off-line learning stage, but it will not add computational burden in the online recognition stage. We will compare the empirical running time of the competing methods in Section 5.3.

\subsection{Experiments on the Multi-PIE expression database}

In this section, we use the large-scale Multi-PIE [49] to verify the effectiveness and efficiency of MBC without considering dimensionality reduction. All the 249 subjects in Session 1 are used as the training set in this experiment. To make the FR more challenging, four subsets with both illumination and expression variations in Sessions 1, 2 and 3 are used for testing. For the training set, we use the 7 frontal images with extreme illuminations $\{0,1,7,13,14,16,18\}$ and neutral expression (refer to Fig. 8(a) for examples). For the testing set, 4 typical frontal images with illuminations $\{0,2,7,13\}$ and different expressions (smile in Sessions 1 and 3, squint and surprise in Session 2) are used (refer to Fig. 8(b) for examples with surprise in Session 2, Fig. 8(c) for examples with squint in Session 2, Fig. 8(d) for examples with smile in Session 1, and Fig. 8(e) for examples with smile in Session 3). We crop and normalize the images to $100 \times 82$. Here the programming environment is Matlab version R2011a. The desktop used is of 1.86 GHz CPU and with 2.99G RAM. 


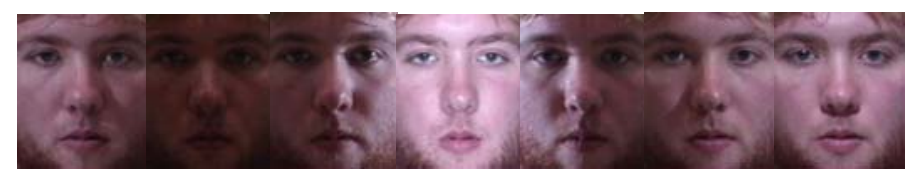

(a)

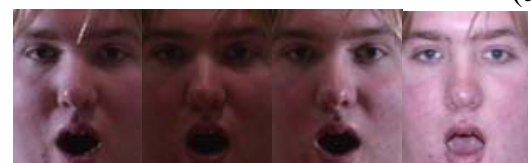

(b)

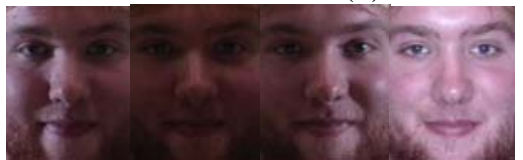

(d)

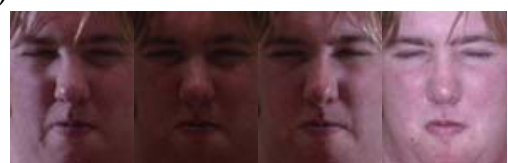

(c)

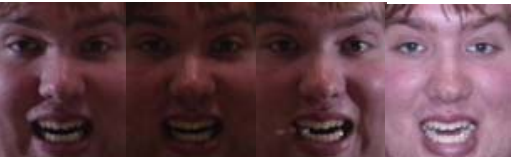

(e)

Figure 8: A subject in Multi-PIE database. (a) Training samples with only illumination variations. (b) Testing samples with surprise expression and illuminations in Session 2. (c) Testing samples with squint expression and illuminations in Session 2. (d) and (e) show the testing samples with smile expression and illumination variations in Session 1 and Session 3, respectively.

Table 3: Face recognition rates (\%) and average running time (second) on the Multi-PIE expression database.

\begin{tabular}{llllll}
\hline Method & Smile-S1 & Smile-S3 & Surprise-S2 & Squint-S2 & Running Time \\
\hline LBP & 96.9 & 68.4 & 68.8 & 76.8 & $\mathbf{0 . 3 1 3}$ \\
LGBP & $\mathbf{9 9 . 2}$ & 77.7 & 72.0 & $\mathbf{8 3 . 4}$ & 12.702 \\
HGPP & $\mathbf{9 9 . 2}$ & 80.3 & $\mathbf{7 5 . 2}$ & $\mathbf{8 3 . 4}$ & 27.532 \\
LGXP & $\mathbf{9 9 . 2}$ & 77.7 & 72.4 & $\mathbf{8 3 . 4}$ & 12.236 \\
MBC-A & $\mathbf{9 9 . 2}$ & $\mathbf{8 5 . 8}$ & 72.9 & $\mathbf{8 3 . 4}$ & 1.016 \\
MBC-O & 99.0 & 80.6 & 72.9 & $\mathbf{8 3 . 4}$ & 1.072 \\
MBC-P & 99.1 & 78.2 & 73.2 & 81.7 & 1.025 \\
\hline
\end{tabular}

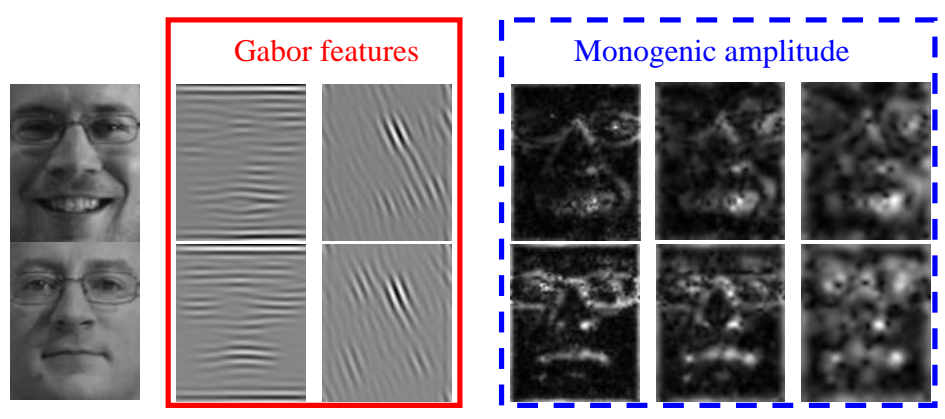

(a)

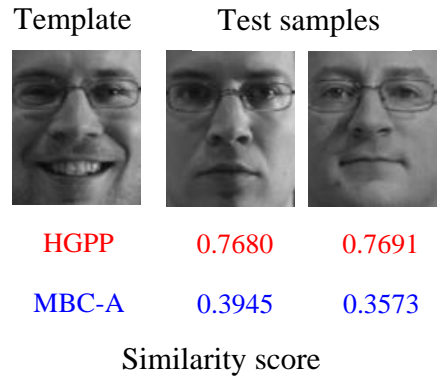

(b)

Figure 9: (a) Examples of Gabor feature and monogenic amplitude feature maps. (b) The similarity scores by HGPP and MBC-A, where the left image is a template image, the middle image is a test image which is from the same class as the template image, and the right test image is from a different class.

Here LBP [8] is used as the baseline method, and we compare the proposed MBC with the state-of-theart methods, such as LGBP [9], HGPP [11] and LGXP [22]. Table 3 lists the face recognition rates and average running time of all the competing methods. It is clear to see that LBP is the fastest one, but with the worst recognition rate. The proposed MBC has very competitive recognition rates as LGBP, HGPP and LGXP. Especially for MBC-A, it has the highest recognition rates in the testing sets of "smile-S1", "smile- 
S3" and "squint-S2". The running time of MBC is about 1 second for each image, less than 1/10 times of that of LGBP, HGPP and LGXP. It is noted that HGPP has the longest running time. The running time results are very consistent with our analysis in Section 5.2.

In this experiment, it can be seen that MBC-A outperforms LGBP and LGXP in all cases. Particularly, MBC-A outperforms much LGBP, HGPP and LGXP on Smile-S3. This is because that on the Smile-S3 dataset, the Gabor features from different subjects may not have enough discrimination. Fig. 9(a) shows some examples of Gabor feature and monogenic amplitude feature maps. One can see that the Gabor feature maps of the two images, which are from two different classes, are very similar, while the monogenic amplitude feature maps of them can have clear difference. Fig. 9(b) shows the similarity scores between two test images and a template image by HGPP and MBC-A. We can see that MBC-A could lead to the correct classification, but Gabor feature based HGPP will lead to a misclassification. Note that the MBC-A similarity score values are usually much lower than those of HGPP because the histogram feature of MBC-A is sparser than that of HGPP. However, this does not reduce the discrimination capability of MBC-A.

\subsection{Experiments on the FERET database}

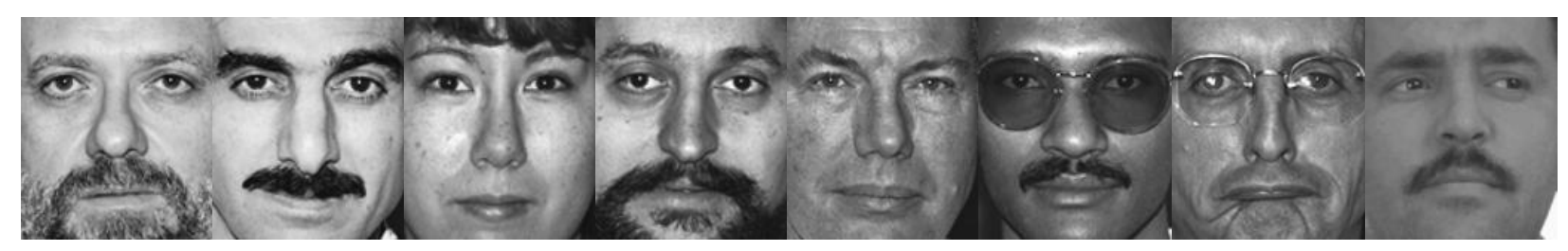

Figure 10: Some samples of normalized face images of FERET.

The FERET database [14][41] is often used to validate an algorithm's effectiveness because it contains many kinds of image variations. By taking Fa subset as a gallery, the probe subsets Fb and Fc are captured with expression and illumination variations. Especially, DupI and DupII consist of images taken at different times. For some people, the time intervals are more than two years between the gallery set and DupI or DupII set. The facial portion of each image is cropped based on the locations of eyes. The cropped face images are then normalized to $150 \times 130$ pixels. Fig. 10 shows some examples of normalized face images. Here the projection matrix of BFLD is learnt from the training set which has 1,002 frontal images from 429 subjects and we set the number of blocks as $M_{b}=5$. 
Table 4: Recognition rates (\%) by using local variation code only and by using the combination of local variation code and imagery intensity code on the $\mathrm{Fb}$ and Dup1 probe sets.

\begin{tabular}{llll}
\hline \multirow{2}{*}{ Method } & $\begin{array}{l}\text { Local } \\
\text { variation code }\end{array}$ & $\begin{array}{l}\text { Local variation \& } \\
\text { imagery intensity code }\end{array}$ \\
\hline \multirow{4}{*}{ Fb } & MBC-A & 96.3 & $\mathbf{9 8 . 1}$ \\
& MBC-A + BFLD & 99.2 & $\mathbf{9 9 . 4}$ \\
& MBC-P & 95.1 & $\mathbf{9 6 . 6}$ \\
& MBC-P + BFLD & 99.0 & $\mathbf{9 9 . 6}$ \\
& MBC-O & 96.4 & $\mathbf{9 7 . 6}$ \\
MBC-O + BFLD & 99.2 & $\mathbf{9 9 . 6}$ \\
\hline \multirow{6}{*}{ DupI } & MBC-A & 66.9 & $\mathbf{7 4 . 4}$ \\
& MBC-A + BFLD & 81.4 & $\mathbf{8 8 . 8}$ \\
& MBC-P & 79.5 & $\mathbf{8 1 . 6}$ \\
& MBC-P + BFLD & 89.2 & $\mathbf{9 2 . 9}$ \\
& MBC-O & 80.3 & $\mathbf{8 5 . 2}$ \\
MBC-O + BFLD & 90.9 & $\mathbf{9 3 . 6}$ \\
\hline
\end{tabular}

In order to illustrate that the combination with local imaginary intensity code could improve much the local variation code, in Table 4 we report the recognition rates by using local variation code only and by using the combination of local variation code and local intensity code on the probe sets of Fb and DupI. It can be clearly seen that in all the tests, the latter one outperforms much the former one. Without BFLD, the combination with local intensity code could improve in average $4.6 \%$ the recognition rate for MBC-A, $1.8 \%$ for MBC-P, and 3\% for MBC-O, respectively. With BFLD, the combination with local intensity code could still improve in average 3.8\% for MBC-A, 2.2\% for MBC-P, and 1.5\% for MBC-O, respectively. This shows that the local imaginary code could provide complementary information to the local variation code.

Table 5 lists the FR results by each individual MBC (e.g., MBC-A, MBC-P or MBC-O), their combinations with BFLD, and the fused MBC (MBC-F). Meanwhile, we give the results by LGBP and LGBP+BFLD reported in [22] as the baseline methods. From Table 5, we can see that all the proposed monogenic codes, MBC-A, MBC-P or MBC-O, could achieve higher recognition rate than LGBP, especially with the biggest improvement of about $11 \%$ in DupI and DupII for MBC-O. BFLD could improve the performance of all methods. When combined with BFLD, MBC-A, MBC-P or MBC-O could achieve higher recognition rates than the method LGBP+BLFD in most cases. In this experiment, the weight $w$ in MBC-F is set to 0.45 since the amplitude component performs a little worse than the monogenic phase part.

The FR results of MBC-F and other state-of-the-art LSF-FR methods, including the feature fusion version of LGXP [22], Tan's method in [26], Zou's method in [27], HGPP [11], LGBP [9] and LBP [8], are compared in Table 6. It can be seen that the proposed MBC-F is the best one for Fb and Fc. In DupI and 
DupII, the recognition rate of MBC-F is slightly lower than the method in [22]. However, it should be noted that our proposed MBC-F method has much lower time and space complexity than the method in [22]. Therefore, the overall performance of MBC-F is better.

Table 5: Recognition rates (\%) of the proposed MBC methods on the FERET probe sets.

\begin{tabular}{lllll}
\hline Method & \multicolumn{4}{c}{ FERET probe sets } \\
& Fb & Fc & DupI & DupII \\
\hline LGBP (Baseline) & 96.0 & 96.0 & 74.0 & 70.0 \\
MBC-A & $\mathbf{9 8 . 1}$ & 97.4 & 74.4 & 72.2 \\
MBC-P & 96.6 & $\mathbf{9 9 . 0}$ & 81.6 & 78.2 \\
MBC-O & 97.6 & $\mathbf{9 9 . 0}$ & $\mathbf{8 5 . 2}$ & $\mathbf{8 3 . 3}$ \\
\hline LGBP +BFLD (Baseline) & 99.0 & 99.0 & 91.0 & 91.0 \\
MBC-A+BFLD & 99.4 & 99.0 & 88.8 & 86.3 \\
MBC-P+BFLD & $\mathbf{9 9 . 6}$ & $\mathbf{9 9 . 5}$ & 92.9 & $\mathbf{9 1 . 0}$ \\
MBC-O+BFLD & $\mathbf{9 9 . 6}$ & $\mathbf{9 9 . 5}$ & $\mathbf{9 3 . 6}$ & 90.2 \\
\hline MBC-F & $\mathbf{9 9 . 7}$ & $\mathbf{9 9 . 5}$ & $\mathbf{9 3 . 6}$ & $\mathbf{9 1 . 5}$ \\
\hline
\end{tabular}

Table 6: Recognition rates (\%) of different methods on the FERET face database.

\begin{tabular}{lllll}
\hline Methods & \multicolumn{4}{c}{ FERET probe sets } \\
& Fb & Fc & Dup I & Dup II \\
\hline Xie’s method in [22] & 99.0 & 99.0 & $\mathbf{9 4 . 0}$ & $\mathbf{9 3 . 0}$ \\
Tan's method in [26] & 98.0 & 98.0 & 90.0 & 85.0 \\
Zou's method in [27] & 99.5 & 99.5 & 85.0 & 79.5 \\
HGPP [11] & 97.5 & 99.5 & 79.5 & 77.8 \\
LGBP [9] & 98.0 & 97.0 & 74.0 & 71.0 \\
LBP [8] & 97.0 & 79.0 & 66.0 & 64.0 \\
MBC-F & $\mathbf{9 9 . 7}$ & $\mathbf{9 9 . 5}$ & $\mathbf{9 3 . 6}$ & 91.5 \\
\hline
\end{tabular}

\subsection{Experiments on the FRGC database}

FRGC 2.0 [13] is a very large scale face database, which consists of 50,000 recordings divided into training and validation partitions. The FRGC challenge problem consists of six experiments, which evaluate FR performance on various conditions, e.g., controlled or uncontrolled lighting and background, 3D imagery, and multi-still imagery [13]. There are three masks, which could be used to generate three receiver operator characteristic (ROC) curves. ROC1 could verify FR on the images collected within semesters, while ROC2 and ROC3 evaluate FR on the images collected within a year and between semesters, respectively. 


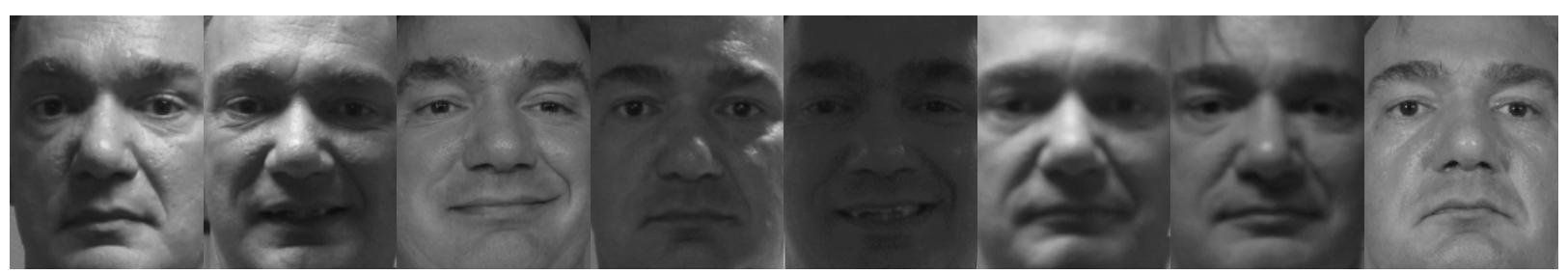

Figure 11: Some samples of normalized face images of FRGC 2.0.

In our experiments, we learn the projection matrix of BFLD based on the given training set $(12,776$ images of 222 persons) and implement our tests on the $1^{\text {st }}$ and $4^{\text {th }}$ experiments, denoted by Exp. 1 and Exp.4. Exp.4 is much more difficult than Exp.1 since the query images in Exp.4 are taken under uncontrolled environment. All the face images are cropped based on the manually located eye centers provided by the original database and normalized to $168 \times 128$ pixels. Some samples are shown in Fig. 11 . Here $M_{r}$ is set as 4 . In order for a stable and effective discriminative feature extraction by dimensionality reduction, BFLD usually requires that the input should have more features. For the feature of monogenic signal representation, the local amplitude (i.e., $A_{\mathrm{lg}}$ in Eq. (10)) is a compact composition of three partial features (i.e., $f_{\mathrm{lg}}, f_{\mathrm{lg}-x}$, and $f_{\text {lg-y }}$ in Eq.(10)). In order to capture more information in amplitude, for MBC-A+BFLD in Exp4, instead of directly encoding on the amplitude, we first encode the local variations of $f_{\mathrm{lg}}, f_{\mathrm{lg}-x}$, and $f_{\mathrm{lg}-y}$, and then in each block we concatenate the three kinds of histogram features. Finally we apply BFLD to the concatenated higher dimensional histogram feature. Although a higher dimensional histogram feature of monogenic amplitude is generated, the time and space complexity is only slightly increased, and the dimension of the final discriminative feature generated by BFLD does not increase.

Similar to Table 5, we list the verification rates of different MBC based methods when the FAR is $0.1 \%$ in Table 7. It can be seen that MBC could achieve much better performance than LGBP in most cases. However, for Exp.4, all MBC and LGBP methods have low validation rates, not larger than 20\%. When BLFD is used to extract the discriminative features, all the methods have great improvements, e.g., more than $60 \%$ for MBC-A and LGBP. We could also see that MBC+BLFD, especially MBC-A+BFLD, has very competing performance with LGBP but with much lower complexity. The ROC3 curves of MBC+BFLD and MBC-F are plotted in Fig. 12, which clearly show that MBC-F could improve the final performance by fusing the complementary monogenic information. 
The recognition results of MBC-F and other state-of-the-art methods, including the fusion version of LGXP [22], Tan's method in [26], Huang's method in [44], Liu's method in [45], Liu's method in [46], Su's method in [15], are listed in Table 8. In the table, all the results for the comparison are directly cited from the related papers. We can see that MBC-F achieves very close results to the best results reported in [15] and [22]. However, considering that both Xie's method in [22] and Su's method in [15] adopt multi-scale and multi-orientation Gabor transformation to generate features, MBC-F has much lower complexity than them, which further validates the effectiveness of the proposed techniques.

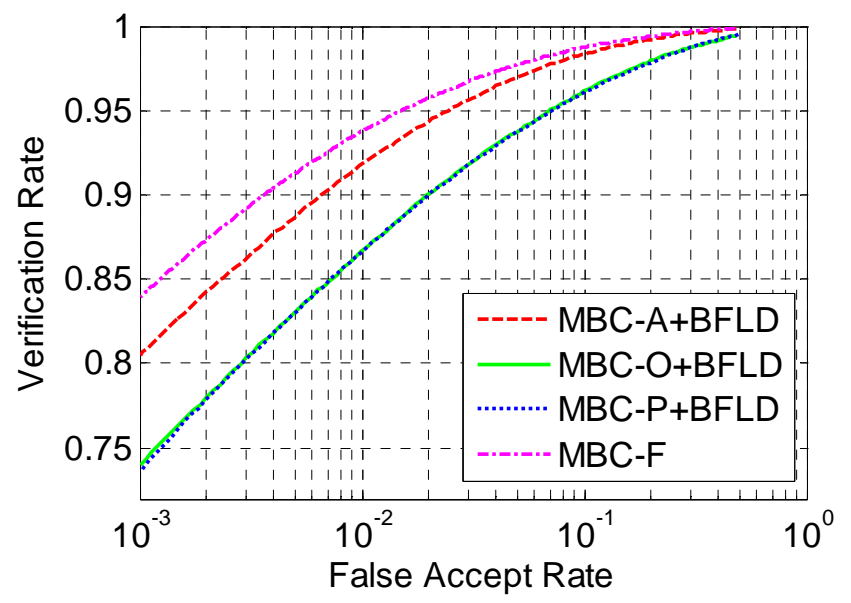

Figure 12: ROC3 curves of different methods on Exp4 of FRGC 2.0 databases.

Table 7: Verification rates by the proposed MBC methods on FRGC 2.0 when FAR $=0.1 \%$.

\begin{tabular}{lllllll}
\hline Method & \multicolumn{3}{c}{ Exp.1 } & \multicolumn{3}{c}{ Exp.4 } \\
& ROC1 & ROC2 & ROC3 & ROC1 & ROC2 & ROC3 \\
\hline LGBP (baseline) & 80.3 & 76.3 & 71.9 & 11.3 & 11.4 & 11.7 \\
MBC-A & 83.8 & 80.4 & 76.7 & 11.0 & 11.5 & 12.1 \\
MBC-P & $\mathbf{8 8 . 0}$ & $\mathbf{8 5 . 0}$ & $\mathbf{8 2 . 0}$ & 16.2 & 16.3 & 16.8 \\
MBC-O & 87.7 & $\mathbf{8 5 . 0}$ & 81.8 & $\mathbf{1 7 . 4}$ & $\mathbf{1 7 . 7}$ & $\mathbf{1 8 . 4}$ \\
\hline LGBP+BFLD (baseline) & $\mathbf{9 8 . 1}$ & $\mathbf{9 7 . 5}$ & $\mathbf{9 6 . 8}$ & $\mathbf{7 9 . 6}$ & $\mathbf{8 0 . 3}$ & $\mathbf{8 1 . 0}$ \\
MBC-A+BFLD & 97.6 & 96.9 & 96.2 & 79.3 & 79.9 & 80.5 \\
MBC-P+BFLD & 97.8 & 97.0 & 96.3 & 71.6 & 72.5 & 73.6 \\
MBC-O+BFLD & 98.0 & 97.4 & $\mathbf{9 6 . 8}$ & 71.8 & 72.8 & 74.0 \\
\hline MBC-F & $\mathbf{9 8 . 5}$ & $\mathbf{9 8 . 0}$ & $\mathbf{9 7 . 5}$ & $\mathbf{8 2 . 1}$ & $\mathbf{8 3 . 0}$ & $\mathbf{8 3 . 9}$ \\
\hline
\end{tabular}

Table 8: Verification rates (\%) by different methods on FRGC 2.0 face database.

\begin{tabular}{llc}
\hline Methods & \multicolumn{2}{c}{ FRGC 2.0 } \\
& Exp.1 & Exp.4 \\
\hline Xie’s method in [22] & 97.5 & 84.9 \\
Tan's method in [26] & N/A & 83.6 \\
Huang's method in [44] & 91.5 & 74.3 \\
Liu's method in [45] & 92.0 & 76.0 \\
Liu's method in [46] & N/A & 81.3 \\
Su's method in [15] & $\mathbf{9 8 . 0}$ & $\mathbf{8 6 . 0}$ \\
MBC-F & 97.5 & 83.9 \\
\hline
\end{tabular}




\subsection{Experiments on the PolyU NIR database}

The PolyU-NIR face database [28] is a large scale near-infrared face database, consisting of 350 subjects, each subject providing about 100 samples. Various variations of face images, such as expression, pose, scale, focus, time, are involved in the capturing. In this paper, we do three tests following the experiment setting in [28]. These three subsets are Exp.1 (419 training samples, 574 gallery samples and 2,762 probe samples), Exp.2 (1,876 training samples, 1,159 gallery samples and 4,747 probe samples), and Exp.3 (576 training samples, 951 gallery samples and 3,648 probe samples). Some samples of Exp.3 are shown in Fig. 13. Because the face images are normalized to $64 \times 64$ pixels, which are much smaller than that of Multi-PIE, FERET and FRGC, we set $\lambda_{\min }=0.55, M_{b}=2$ and the dimensionality of discriminative feature as 100 .

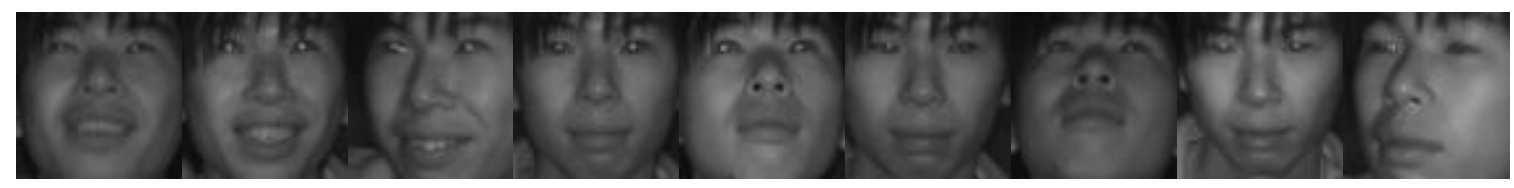

Figure 13: Some samples of normalized face images of Exp.3 on the PolyU NIR database.

Table 9 lists the recognition rates by MBC methods and the baseline method, Gabor-DBC, proposed in [28]. From Table 9, we can see that all the MBC methods are better than the baseline method; and BFLD could further improve MBC's performance. The recognition results of MBC-F and other state-of-the-art results reported in [28] are shown in Table 10. It can be seen that the proposed MBC-F outperforms the second best method by 1.2\% in Exp.1, 2.2\% in Exp.2 and 2.8\% in Exp.3. Meanwhile, compared to these Gabor feature based methods, the proposed MBC methods have much lower time and space complexity.

Table 9: Recognition rates by the proposed MBC methods on the PolyU-NIR database.

\begin{tabular}{llll}
\hline Methods & \multicolumn{3}{c}{ PolyU-NIR probe sets } \\
& Exp.1 & Exp.2 & Exp.3 \\
\hline \hline Gabor-DBC (baseline) & 97.6 & 92.3 & 92.5 \\
MBC-A & 98.7 & 93.5 & 94.1 \\
MBC-P & 98.3 & 92.3 & 92.7 \\
MBC-O & 98.4 & 92.6 & 93.2 \\
MBC-A + BFLD & 98.6 & 94.0 & 94.8 \\
MBC-P + BFLD & 98.5 & 93.5 & 94.4 \\
MBC-O + BFLD & 98.7 & 93.6 & 94,4 \\
MBC-F & $\mathbf{9 8 . 8}$ & $\mathbf{9 4 . 5}$ & $\mathbf{9 5 . 3}$ \\
\hline
\end{tabular}


Table 10: Recognition rates (\%) by different methods on the PolyU-NIR probe sets.

\begin{tabular}{llll}
\hline Methods & \multicolumn{3}{c}{ PolyU-NIR } \\
& Exp.1 & Exp.2 & Exp.3 \\
\hline Gabor-PCA & 82.4 & 90.2 & 60.8 \\
Gabor-LDA & 91.8 & 92.1 & 82.4 \\
LGBP & 95.1 & 89.3 & 89.8 \\
Gabor-DBC & 97.6 & 92.3 & 92.5 \\
MBC-F & $\mathbf{9 8 . 8}$ & $\mathbf{9 4 . 5}$ & $\mathbf{9 5 . 3}$ \\
\hline
\end{tabular}

\subsection{Discussions}

From the above complexity analysis and experimental results, it can be seen that the proposed MBC has competitive accuracy with or higher accuracy than the Gabor feature based methods (e.g., LGBP [9], HGPP [11], and LGXP [22]), while it has much lower cost (e.g., less than 1/10 times running time on the MPIE database). This merit of MBC comes from two parts: the power of monogenic signal representation and the effectiveness of MBC’s pattern coding strategy.

Like Gabor wavelet representation, monogenic signal representation can effectively extract the discrimination information embedded in the original signal by decoupling the local energy (local amplitude) and structure (local phase and local orientation). Different from Gabor features, which are extracted along several orientations by using steerable filters, in monogenic signal representation the local orientation is the main orientation of the linear structure with large support, and the local phase describes the structure along the main orientation [12]. This strategy of extracting only the information along the dominant orientation is somewhat similar to the maximum-like pooling mechanism in the visual processing in cortex [50], and it leads to a compact but effective representation of the image. Our experimental results validate that monogenic signal analysis is very suitable for face image representation, probably because human facial local structures can be well characterized along one dominant orientation. In addition, the adaptation of logGabor filters in multi-scale monogenic signal representation makes it efficient in capturing the multi-scale information.

The promising performance of MBC also comes from the proposed binary coding scheme, which is well suited to describe the monogenic signal representation. In MBC, we not only encode the local variation of monogenic amplitude and monogenic phase, but also encode the monogenic local imagery intensity to provide complementary information to the local variation. As shown in Table 4, this coding strategy could 
exploit more useful information of monogenic signal representation. In addition, the three parts of MBC:

MBC-A, MBC-P and MBC-O, encode the energetic, structural and geometric information of a 2D signal, respectively, which are complementary to each other. The experimental results show that MBC on monogenic amplitude, orientation and phase could individually achieve high recognition rate on different datasets. More importantly, a strategy by using a weighted average to fuse these three similarity scores (e.g., MBC-A, MBC-P and MBC-O) could further improve the recognition accuracy and achieve state-of-the-art FR performance.

\section{Conclusion}

We proposed a novel face representation model, namely monogenic binary coding (MBC), based on the monogenic signal representation. One of the best merits of MBC is that it has much less time and space complexity than the widely used Gabor transformation based local feature extraction method. Through multi-scale monogenic signal representation, three kinds of features (e.g., local amplitude, local orientation and local phase) can be generated, and then encoded by the proposed monogenic local variation coding and monogenic imagery intensity coding procedures. The produced MBC pattern maps are used to compute the statistical features (e.g., histogram), which are then used to measure the similarity for face recognition. The extensive experiments on the benchmark face databases, including FERET, FRGC 2.0, Multi-PIE and PolyU-NIR, clearly showed that the proposed MBC methods not only have significantly lower time and space complexity than the state-of-the-art Gabor feature based face recognition methods, but also have very competitive or even better recognition rates.

\section{References}

[1] W.Y. Zhao, R. Chellppa, P.J. Phillips, and A. Rosenfeld, "Face recognition: A literature survey," $A C M$ Computing Survey, vol. 35, no. 4, pp. 399-458, 2003.

[2] N. Poh, C.H. Chan, J. Kittler, S. Marcel, C.M. Cool, E.A. Rúa, J.L.A. Castro, M. Villegas, R. Paredes, V. Struc, N. Pavesic", A.A. Salah, H. Fang, and N. Costen, "An Evaluation of Video-to-Video Face Verification," IEEE Trans. Information Forensics and Security, Vol. 5, No. 4, pp. 781-801, 2010.

[3] Z.F Li, U. Park, and A.K. Jain, “A Discriminative Model for Age Invariant Face Recognition,” IEEE Trans. Information Forensics and Security, Vol. 6, No. 3, pp. 1028-1037, 2011.

[4] H.B. Ling, S. Soatto, N. Ramanathan, and D.W. Jacobs, "Face Verification Across Age Progression Using Discriminative Methods," IEEE Trans. Information Forensics and Security, Vol. 5, No. 1, pp. 82-91, 2010.

[5] M. Turk and A. Pentland, "Eigenfaces for recognition,” Journal of Cognitive Neuroscience, vol. 13, no. 1, pp. 7186, 1991. 
[6] P. Belhumeur, J. Hespanha, and D. Kriegman, "Egienfaces vs. fisherfaces: Recognition using class specific linear projection,” IEEE Trans. Pattern Anal. Machine Intell., vol. 19, no. 7, pp. 711-720, 1997.

[7] C. Liu and H. Wechsler, "Gabor feature based classification using the enhanced Fisher linear discriminant model for face recognition,” IEEE Trans. Image Processing, vol. 11, no. 4, pp. 467-476, 2002.

[8] A. Timo, H. Abdenour, and P. Matti, "Face recognition with Local Binary Patterns," Proc. European Conf. Computer Vision, 2004.

[9] W. Zhang, S. Shan, W. Gao, X. Chen, and H. Zhang, "Local Gabor binary pattern histogram sequence (LGBPHS): A novel non-statistical model for face representation and recognition,” Proc. IEEE Int'l Conf. Computer Vision, 2005.

[10] W. Zhang, S. Shan, X. Chen, and W. Gao, “Are Gabor Phases Really Useless for Face Recognition?” Proc. Int'l Conf. Pattern Recognition, 2006.

[11] B. Zhang, S. Shan, X. Chen, and W. Gao, "Histogram of Gabor Phase Patterns (HGPP): A Novel Object Representation Approach for Face Recognition,” IEEE Trans. Image Processing, vol. 16, no. 1, pp. 57-68, 2006.

[12] M. Felsberg and G. Sommer, “The monogenic signal,” IEEE Trans. Signal Processing, vol. 49, no. 12, pp. 31363144, 2001.

[13] P.J. Phillips, P.J. Flynn, T. Scruggs, K. W. Bowyer, J. Chang, K. Hoffman, J. Marques, J. Min, and W. Worek. "Overview of the Face Recogniton Grand Challenge," Proc. IEEE Int'l Conf. Computer Vision and Pattern Recognition, 2005.

[14] P.J. Phillips, H. Moon, S.A. Rizvi, and P.J. Rauss, "The FERET Evaluation Methodology for Face Recognition Algorithms,” IEEE Trans. Pattern Anal. Mach. Intell., Vol. 22, pp. 1090-1104, 2000.

[15] Y. Su, S. Shan, X. Chen, and W. Gao, "Hierarchical Ensemble of Global and Local Classifiers for Face Recognition,” Proc. Int'l Conf. Computer Vision, 2007.

[16] T. Ojala, M. Pietikäinen, and D. Harwood, “A comparative study of texture measures with classification based on feature distributions,” Pattern Recognition, vol. 29, no. 1, pp. 51-59, 1996.

[17] J. Yang and C.J. Liu, "Horizontal and Vertical 2DPCA-Based Discriminant Analysis for Face Verification on a Large-Scale Database,” IEEE Trans. Information Forensics and Security, Vol. 2, No. 4, pp. 781-792, 2007.

[18] S. Venkatesh and R. Owens, “On the classification of image features," Pattern Recognition Letters, vol. 11, pp. 339-349, 1990.

[19] E. Stein and G. Weiss. Introduction to Fourier analysis on Euclidean Spaces. Princeton, NJ: Princeton Univ. Press, 1971.

[20] D. Fields, "Relations between the statistics of natural images and the response properties of cortical cells," Journal of Optical Society of America, vol. 4, no. 12, pp. 2379-2394, 1987.

[21] G. H. Granlund and H. Knutsson, Signal Processing for Computer Vision, Kluwer Academic Publishers, 1995.

[22] S.F. Xie, S.G. Shan, X.L. Chen, and J. Chen, "Fusing Local Patterns of Gabor Magnitude and Phase for Face Recognition,” IEEE Trans. Image Processing, vol. 19, no. 5, pp. 1349-1361, 2010.

[23] M. Yang, L. Zhang, L. Zhang and D. Zhang, "Monogenic Binary Pattern (MBP): A Novel Feature Extraction and Representation Model for Face Recognition,” Proc. Int'l Conf. Pattern Recognition, 2010.

[24] H.K. Ekenel, H. Gao, and R. Stiefelhagen, "3-D Face Recognition Using Local Appearance-Based Models," IEEE Trans. Information Forensics and Security, Vol. 2, No. 3, pp. 630-636, 2007.

[25] Z.H. Guo, L. Zhang, and D. Zhang, "A completed modeling of local binary pattern operator for texture classification,” IEEE Trans. Image Processing, vol. 19, no. 6, pp. 1657-1663, 2010.

[26] X. Tan and B. Triggs, "Fusing Gabor and LBP feature sets for kernel-based face recognition," IEEE International Workshop on analysis and modeling of faces and gestures, 2007.

[27] J. Zou, Q. Ji, and G. Nagy, “A comparative study of local matching approach for face recognition,” IEEE Trans. Image Processing, vol. 16, no. 10, pp. 2617-2628, 2007.

[28] B.C. Zhang, L. Zhang, D. Zhang, and L.L. Shen, "Directional binary code with application to PolyU near-infrared face database,” Pattern Recognition Letters, vol. 31, no. 14, pp. 2337-2344, 2010.

[29] J. Wright, A. Yang, A. Ganesh, S. Sastry, and Y. Ma, "Robust face recognition via sparse representation,” IEEE Trans. Pattern Anal. Machine Intell., vol. 31, no. 2, pp. 210-227, 2009.

[30] M. Yang and L. Zhang, "Gabor Feature based Sparse Representation for Face Recognition with Gabor Occlusion Dictionary,” Proc. European Conf. Computer Vision, 2010.

[31] M. Felsberg and G. Sommer, "The monogenic scale-space: A unifying approach to phase-based image processing in scale-space,” J. Math. Imag. Vis., vol. 21, no. 1, pp. 5-26, 2004.

[32] M. Unser, D. Sage, and D.V.D. Ville, "Multiresolution monogenic signal analysis using the riesz-laplace wavelet transform,” IEEE Trans. Image Processing, vol. 18, no. 11, pp. 2402-2418, 2009.

[33] L. Zhang, L. Zhang, Z.H. Guo, and D. Zhang, "Monogenic-LBP: A new approach for rotation invariant texture classification,” Proc. Int'l Conf. Pattern Recognition, 2010.

[34] S. V. Fischer, F. Sroubek, L, Perrinet, R. Redondo, and G. Cristobal, "Self-Invertible 2D Log-Gabor Wavelet," International Journal of Computer Vision, vol. 75, no. 2, pp. 231-246, 2007.

[35] M. Yang, L. Zhang, J. Yang and D. Zhang, “Robust sparse coding for face recognition,” Proc. IEEE Int'l Conf. Computer Vision and Pattern Recognition, 2011. 
[36] X.Tan and B.Triggs, "Enhanced Local Texture Feature Sets for Face Recognition under Difficult Lighting Conditions,” IEEE Trans. Image Processing, vol. 19, no. 6, 1635-1650, 2010.

[37] X. He, S. Yan, Y. Hu, P. Niyogi, and H.J. Zhang, "Face recognition using laplacianfaces”, IEEE Trans. Pattern Anal. Machine Intell., vol. 27, no. 3, 328-340, 2005.

[38] T. Inan, U. Halici, “3D Face Recognition with Local Shape Descriptors,” IEEE Trans. Information Forensics and Security, vol. 7, no. 2, pp. 577-587, 2012.

[39] Z. Zhou, A. Wagner, H. Mobahi. J. Wright, and Y. Ma, "Face recognition with contiguous occlusion using markov random fields,” Proc. IEEE Int'l Conf. Computer Vision, 2009.

[40] E. Elhamifar and R. Vidal, "Robust classification suing structured sparse representation,” Proc. IEEE Int'l Conf. Computer Vision and Pattern Recognition, 2011.

[41] P.J. Phillips, H. Wechsler, J. Huang, and P. Rauss, "The FERET database and evaluation procedure for face recognition algorithms,” Image and Vision Computing, Vol. 16, No. 5, pp. 295-306, 1998.

[42] J. Cook, V. Chandran, S. Sridharan and C. Fookes, "Gabor Filter Bank Representation for 3D Face Recognition,” Proc. Digital Imaging Computing: Techniques and Applications, 2005.

[43] P. Kovesi, "Invariant Measures of Image Features from Phase Information,” Ph.D. thesis, The University of Western Australia, 1996.

[44] W. Huang, G. Park, and J. Lee, "Multiple Face Model of Hybrid Fourier Feature for Large Face Image Set”, Proc. IEEE Int'l Conf. Computer Vision and Pattern Recognition, 2006.

[45] C. Liu, "Capitalize on Dimensionality Increasing techniques for Improving Face Recognition Grand Challenge Performance,” IEEE Trans. Pattern Anal. Machine Intell., vol. 28, no. 5, pp. 725-737, 2006.

[46] Z. Liu and C. Liu, "Fusion of the complementary Discrete Cosine Features in the YIQ color space for face recognition”, Computer vision and Image Understanding, vol. 111, no. 3, pp. 249-262, 2008.

[47] J. Cook, C. McCool, V. Chandran and S. Sridharan, "Combined 2D / 3D Face Recognition using Log-Gabor Templates”, Proc. IEEE Int'l Conf. Video and Signal Based Surveillance, 2006.

[48] J. Cook, V. Chandran, and C. Fookes, “3D face recognition using Log-Gabor templates”, Proc. British Machine Vision Conference, 2006.

[49] R. Gross, I. Matthews. J. Cohn, T. Kanade, and S. Baker, “Multi-PIE,” Image and Vision Computing, vol. 28, pp. 807-813, 2010.

[50] M. Riesenhuber and T. Poggio, "Hierarchical models of object recognition in cortex,” Nature neuroscience, vol. 2, no. 11, pp. 1019-1025, 1999. 